\title{
Immersive Interactive Quantum Mechanics for Teaching and Learning Chemistry
}

\author{
Thomas Weymuth ${ }^{\mathrm{a}}$ and Markus Reiher ${ }^{\star}$
}

\begin{abstract}
The impossibility of experiencing the molecular world with our senses hampers teaching and understanding chemistry because very abstract concepts (such as atoms, chemical bonds, molecular structure, reactivity) are required for this process. Virtual reality, especially when based on explicit physical modeling (potentially in real time), offers a solution to this dilemma. Chemistry teaching can make use of advanced technologies such as virtual-reality frameworks and haptic devices. We show how an immersive learning setting could be applied to help students understand the core concepts of typical chemical reactions by offering a much more intuitive approach than traditional learning settings. Our setting relies on an interactive exploration and manipulation of a chemical system; this system is simulated in real-time with quantum chemical methods, and therefore, behaves in a physically meaningful way.
\end{abstract}

Keywords: Haptic devices, Interactive quantum chemistry, Real-time quantum chemistry, Virtual reality

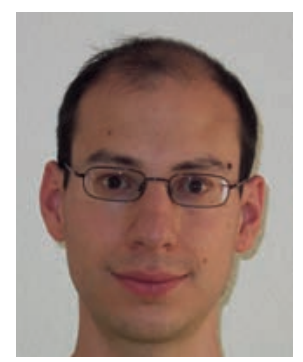

Thomas Weymuth was born in Zurich, Switzerland, in 1987. He joined the research group of Markus Reiher in 2010 and received his $\mathrm{PhD}$ from ETH Zurich in 2013. After working in the private sector for four years, he rejoined the group of Markus Reiher as research associate at ETH in 2017. His research interests include computational spectroscopy and the efficient exploration of chemical reaction networks. He is interested in the development of new and improved teaching environments.

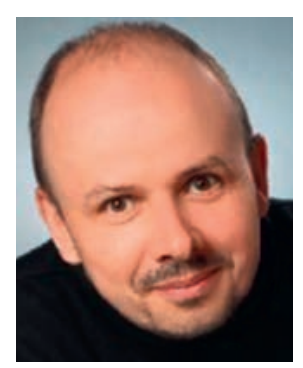

Markus Reiher received his $\mathrm{PhD}$ in theoretical chemistry from the University of Bielefeld in 1998, working in the group of Juergen Hinze. After his habilitation in the group of Bernd Artur Hess at the University of Erlangen, he worked as a private docent in Erlangen in 2003 and at the University of Bonn in 2004. In 2005, he accepted an offer for a professorship in physical chemistry from the University of Jena, where he worked until he moved to ETH Zurich in 2006. His research covers many different areas in theoretical chemistry which range from relativistic quantum chemistry to the development of new electron-correlation theories and smart algorithms for the efficient exploration of complex chemical reaction networks. As a dedicated teacher, he is interested in conveying new ways to help students grasp physical and chemical concepts.

\section{Introduction}

To understand chemistry, students in schools and at universities are exposed to highly abstract ideas and concepts about a tiny molecular world that is elusive to our senses. In fact, it is this inaccessibility of the molecular world that sets chemistry apart from neighboring disciplines such as physics and biology: we understand macroscopic observations of chemical reactions (such as color changes) solely in terms of a dance of atoms at the nanometer scale in the molecular program.

Chemical concepts are introduced and explained by following either an abstract mathematical approach or by a sequence of examples. Whereas the former requires a good understanding of mathematical ideas combined with imaginative capabilities, the latter approach is hampered by the need for advanced pattern recognition capabilities. A viable alternative to such an approach might be offered by explicit physical modeling in virtual reality.

Computer visualizations in videos and alike are often used to display complex dynamical processes like the rearrangements of atoms in a chemical reaction. While there is evidence that such dynamical visualizations are indeed helpful, ${ }^{[1]}$ other studies have shown that movies are often not more effective than static pictures. ${ }^{[2]}$ A possible explanation for this observation is that any dynamical representation is transient in nature, hence requiring the student to remember previously obtained information while simultaneously acquiring new information. The resulting high load on the working memory hinders effective learning. ${ }^{[3]}$ Van Gog et al. argue that especially in cases where human movements are depicted (e.g. a surgeon carrying out a complicated procedure), students can benefit a lot by watching a corresponding dynamical representation. ${ }^{[4]}$ They argue that in such cases, the observation of a human performing a certain task automatically activates the socalled mirror neuron system, which is not activated if the student watches a depiction of a non-human movement. The mirror neurons might lead to a reduced load on the working memory, thereby explaining the effectiveness of videos in such cases. Moreover, it is known that the learning effect of all kinds of dynamical visualizations, also those depicting non-human motions, are enhanced by involving a student's motor system. ${ }^{[3]}$

In chemistry, technologies such as virtual or augmented reality and haptic devices have been introduced but have not found widespread application so far. ${ }^{[5-34]}$ Virtual and augmented reality 
technologies allow a person to be immersed into a virtual world. In virtual reality, the entire world experienced by a person is virtual, whereas in augmented reality (sometimes also called mixed reality), the person experiences the real world while additional virtual objects are added to it, e.g. through a suitable projection on a head-up display. Currently, the immersion into the virtual world is most easily achieved with a headset, which provides a stereoscopic head-mounted display, stereo sound, as well as head motion tracking sensors (see Fig. 1). With this, a person is able to look around in and explore the virtual world. For this to be sensible, it is decisive that reliable physical modeling is used. In addition, the movement of the entire body of a person is often tracked by means of fixed external sensors. This then allows a person to move around freely in the virtual world. Finally, hand-operated controllers are used to interact with the objects of the virtual world. Such a fully immersive, virtual reality holds a great potential to help students understand better a range of chemical phenomena (see, e.g. refs. $[12,29,30,32,33,35-40])$. For example, the structure of a large molecule with a complicated three-dimensional shape can easily be inspected from all possible angles, and the rearrangements of the individual atoms of this molecule as it undergoes a certain chemical reaction can be examined with great scrutiny. ${ }^{[12,20,22-29,31-33,37,39]}$ Since the person experiencing such a

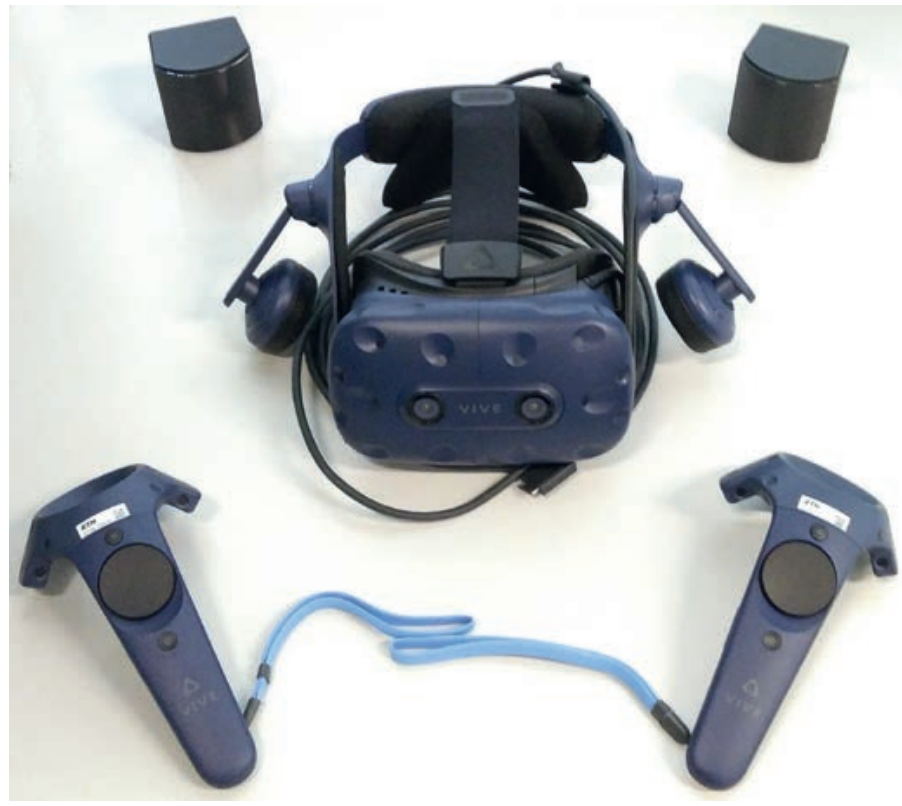

Fig. 1. A virtual reality device (here: 'Vive Pro' from HTC) consisting of a headset (center), two hand-held control sticks (bottom left and right) as well as two base stations used to track a person's position (top left and right).

virtual reality is fully immersed into it, it should provide an ideal environment for teaching, learning, and, of course, research.

Another way to literally create a physical experience and to enhance the human perception of the molecular world is the introduction of haptic devices. A haptic device is a force-feedback hardware device, e.g. one that can transmit a force through electric motors to some hardware attached to them (complex settings may even require one to wear an exoskeleton). The simplest variants that are commercially available comprise pen-like haptic pointers (see Fig. 2 ), which can be moved, rotated, and tilted freely in space. Motors in the joints of the arm of the haptic device can exert a force on the haptic pointer, which is then felt by the person operating the pointer. This force feedback addresses the haptic sense (i.e. the sense of touch) of the person, thereby complementing the visual and auditory senses typically engaged when working with virtual reality such as a molecule displayed on a computer screen. This renders the virtual reality more immersive and improves the intuitiveness with which one can interact with the molecular world. For example, a haptic device allows one to experience the forces acting on a certain atom within a molecule in a very direct and intuitive way. ${ }^{[13]}$ Not surprisingly, previous research has demonstrated that haptic devices can be useful in chemical education. ${ }^{[41-44]}$ Besides this use in chemical education and research, haptic devices are also employed, for example, in healthcare for surgical training, ${ }^{[45-47]}$ and in the graphics industry for three-dimensional modeling. ${ }^{[45,48]}$

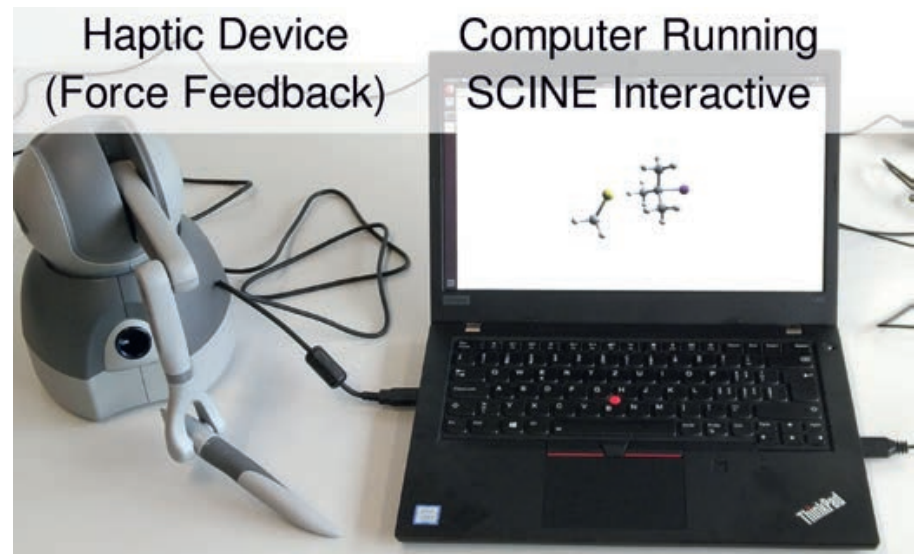

Fig. 2. A haptic pointer device ('Touch' from 3D Systems) on the left (with the haptic pointer being in front) connected to a laptop on the right, set up to interactively explore a chemical reaction.

Irrespective of the way a student or researcher interacts with a virtual chemical system, it needs to behave according to the laws of quantum mechanics which govern the interaction of electrons and nuclei and their dynamics that give rise to its reactive behavior. Only when simulated in a physically reliable way, which guarantees a faithful and accurate representation of the molecular process, the virtual experience will be useful for educational (and research) purposes. Otherwise, students will not be able to reproduce and understand molecular processes as they would be plagued by artefacts and misleading experiences. For a truly immersive learning experience, the physical simulation has to be done in real-time, such that the user is able to manipulate a molecule and experience the consequences of this manipulation without any noticeable delay. This is particularly challenging for a procedure rooted in quantum mechanics (and is the reason why haptics and virtual reality was first explored with classical force fields instead). Due to the ever-increasing performance of modern computers, interactive, real-time quantum chemistry has recently become possible. ${ }^{[13,15-18,21,49]}$ The algorithmic developments made by our research group to accommodate interactive quantum chemistry are assembled in our software package called SCINE Interactive ${ }^{[50]}$ as well as in the program package Samson. ${ }^{[51]}$

This paper demonstrates how chemical concepts can be understood by using haptic devices and interactive quantum chemistry. It is organized as follows: First, we briefly review interactive quantum chemistry and the SCINE Interactive software package, which is available free of charge on our webpage, and highlight their potential role in chemical education. Then, in section 3 , we illustrate the usefulness of this new type of computer-assisted learning by means of a haptic device and real-time quantum simulations at the example of a few prototypical learning tasks. Finally, we provide conclusions and an outlook in section 4 .

\section{The SCINE Interactive Software Package in Chemical Education}

SCINE Interactive ${ }^{[50]}$ is the original implementation of realtime quantum chemistry, a concept invented by us in $2013,{ }^{[16]}$ for 
molecular structure and reactivity exploration with self-consistent orbital optimizations. At the heart of real-time quantum chemistry are ultra-fast electronic structure calculations which deliver quantum chemical results (almost) instantaneously. This then enables a person to explore the potential energy surface of a chemical system in real time, immersively and interactively. The person can manipulate the molecular structure with a mouse or a haptic device and directly perceive the response of the system to this manipulation through visual and also haptic feedback.

For a truly interactive experience, visual feedback needs to be provided at a rate of about $60 \mathrm{~Hz}$, while haptic feedback requires a much higher rate of about $1000 \mathrm{~Hz} \cdot{ }^{[49]}$ Currently, semi-empirical methods are the preferred approach to generate sufficiently reliable energies and forces on a timescale of a few to a few hundred milliseconds. Naturally, real-time quantum chemistry required the development of techniques accelerating electronic structure calculations. ${ }^{[16,18,52]}$ In particular, for the very high refresh rates needed for a haptic device, we introduced a mediator potential which locally approximates the true potential energy surface and which can be evaluated very efficiently. ${ }^{[49]}$ By virtue of these developments, the computing hardware necessary for an immersive interactive experience is far from being demanding. In fact, a standard laptop is sufficient ( $c f$., Fig. 2), even if a haptic device is to be employed.

Being able to explore and manipulate a chemical system interactively and in real time allows one to understand many complicated concepts in an intuitive way. Next, we give specific examples how real-time quantum chemistry, possibly enhanced with a haptic device or a virtual reality framework, can be used for teaching chemistry.

\section{Example Tasks}

\subsection{First Steps}

First, the students are given the Cartesian coordinates of the three nuclei of a squeezed water molecule as an XYZ file. The students should then recognize that with these coordinates, the $\mathrm{H}-\mathrm{O}-\mathrm{H}$ bond angle is $90^{\circ}$, which is not the equilibrium structure of water. Upon loading these coordinates into SCINE Interactive, the students see that the structure promptly relaxes, due to the ultra-fast quantum structure optimization running in the background, by opening up the $\mathrm{H}-\mathrm{O}-\mathrm{H}$ angle until the equilibrium structure is reached.

Another aspect which is slightly more subtle for most students to understand is that the XYZ file contains no information whatsoever about the bonds present in the molecule. Instead, this information is obtained from the quantum mechanical calculations running in the background of the interactive exploration. In fact, bonds are drawn in the graphical user interface based on a simple distance-based measure (that can be supplemented with bond order information taken from the underlying electronic wave function by drawing tubes between atomic spheres of increasing diameter with increasing bond order).

Students not having any prior experience operating a haptic device can use this simple example system to get acquainted with the interactive exploration of a chemical system with force feedback. In particular, they can develop a feeling for how strong a force they apply to an atom can be before the atom gets abstracted from the molecule (note, however, that there is a scaling factor that mediates between the molecular and the macroscopic force): As long as the force is below some value, it will not be possible to abstract the atom from the rest of the molecule; rather, upon a slight displacement of an atom, the other atoms will follow due to a continuously running structure optimization in the background that removes the excess energy in the system, and in effect the entire molecule translates in space. Abstraction of an atom will only be possible if a sufficiently strong force is applied. Once an atom has been abstracted, students will quickly discover that the system has a strong tendency to reassemble to a full water molecule. Only if the abstracted atom is quickly brought to a rather large distance (say, about ten Ångstroms), one finds a quasi-stable system consisting of an $\mathrm{OH}$ radical and a hydrogen atom. From such a situation, one can try to attach the separated hydrogen atom to the other hydrogen atom, rather than to the oxygen atom, effectively forming $\mathrm{H}-\mathrm{H}-\mathrm{O}$. As it turns out, it is virtually impossible to create such a molecule (because the underlying quantum description prevents it and the haptic device allows one to experience and thus learn this fact easily). Whenever one approaches the hydrogen atom of the $\mathrm{OH}$ group, the hydroxy radical rotates and an $\mathrm{O}-\mathrm{H}$ bond is established. Also, when one very carefully approaches the $\mathrm{OH}$ group along the $\mathrm{O}-\mathrm{H}$ bond, any slight (and, in practice, unavoidable) deviation from a perfect linear approach will induce a rotation of the hydroxy radical because of the continuously running structure optimization.

\subsection{Nucleophilic Substitution}

Another instructive example is the prototypical $\mathrm{S}_{\mathrm{N}} 2$ reaction of methanethiolate and 2-iodo-2-methyl propane:

$$
\mathrm{CH}_{3} \mathrm{~S}^{-}+\left(\mathrm{CH}_{3}\right)_{3} \mathrm{CI} \rightarrow\left(\mathrm{CH}_{3}\right)_{3} \mathrm{CSCH}_{3}+\mathrm{I}^{-}
$$

When interactively exploring this reaction, students will quickly find that the reaction occurs readily; it is straightforward to carry out with a haptic device. In fact, without bothering too much about the relative orientation of the two molecules, as soon as the sulfur atom is brought close enough to the tertiary carbon atom of 2-iodo-2-methyl propane, the reaction occurs. One observes the typical pentagonal-bipyramidal transition state, i.e. for a short amount of time, the sulfur atom has already developed a partial bond to the tertiary carbon atom, while the iodine atom is still partially bound to the molecule (Fig. 3).

For this reaction, the overall charge set in the quantum chemical calculations is -1 . It is very instructive to study what happens when the system is considered overall electrically neutral. Even though one has removed only a single electron from the total system, one finds that the reaction is now almost impossible to induce. This is an excellent exercise to understand the role of a good nucleophile and a good leaving group. Only with a very careful, time-consuming operation, the desired end product can be created eventually. It is, however, much more likely that a completely different reaction altogether will be found first, in particular if one is not (yet) so skilled in the operation of a haptic device.

Hence, one immediately understands that $\mathrm{CH}_{3} \mathrm{~S}^{-}$is a good nucleophile, while $\mathrm{CH}_{3} \mathrm{~S}$ is not (naturally, to create this insight for a novice requires a lot of additional explanation, while the real-time exploration transmits it as an immediate puzzle whose explanation can then directly follow within the same setting - by further visualizations such as charge density flow during reaction, energy changes, and so forth). Likewise, $\mathrm{I}^{-}$is a good leaving group, while the neutral iodine atom is not. Using the haptic device, students can experience and literally feel the difference that a single electron can make in almost no time. A movie showcasing this example task can be found on YouTube. ${ }^{[53]}$

\subsection{Electrophilic Aromatic Substitution}

As a last example we consider the interactive exploration of the reaction between aniline and $\mathrm{Br}^{+}$, i.e. an electrophilic aromatic substitution (Fig. 4). We note in passing that also for this reaction care has to be taken to set the correct overall charge. Otherwise, spurious effects such as the ones described in the preceding section occur. When moving the bromine cation close to the benzene ring, students will find that both the ortho- and the para-substituted derivative can be created easily, and both are energetically strongly 
favored; about $230 \mathrm{~kJ} / \mathrm{mol}$ are released in both cases (which may be visualized by a color change of the background). Creating the meta-substituted derivative is also possible without too much dif-
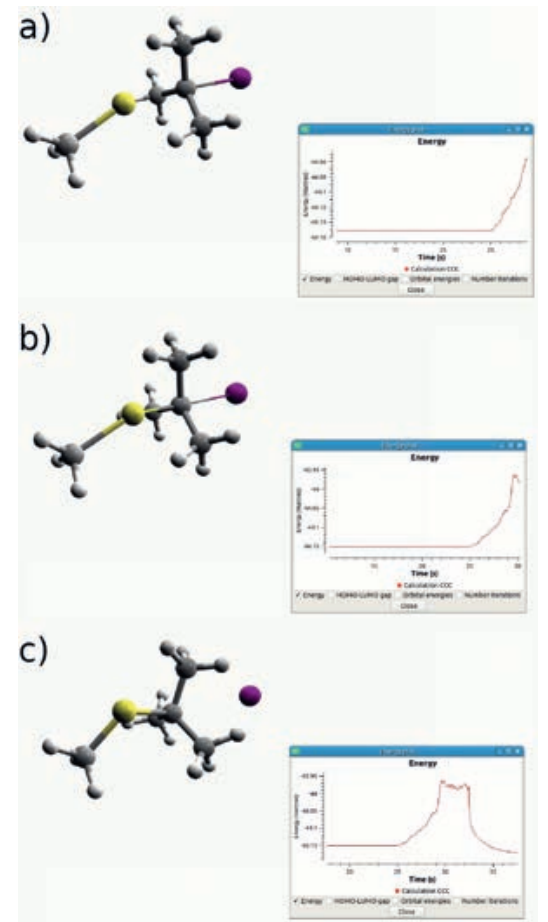

Fig. 3. Consecutive screenshots of the example $S_{N} 2$ reaction. a) The operator moves the methanethiolate molecule towards the reactive center; note how the energy rises. b) Depiction of the approximate transition state. c) The bond to the $\mathrm{I}^{-}$leaving group is fully broken and the system is allowed to relax; note the energy set free in this process. The full movie is available on YouTube. ${ }^{[53]}$

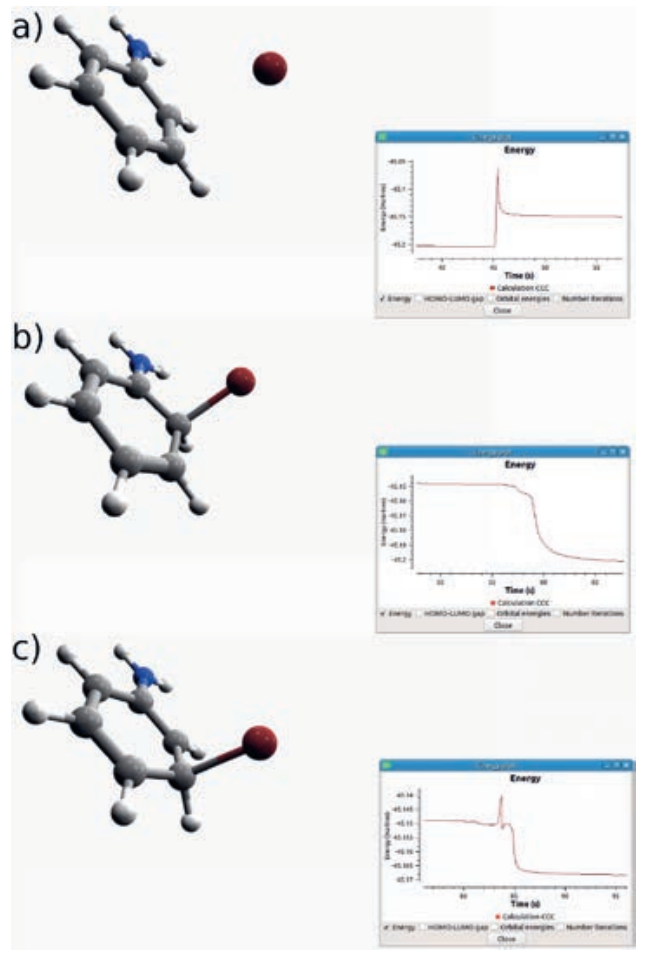

Fig. 4. Consecutive screenshots of the example electrophilic aromatic substitution reaction. a) The operator approaches the benzene ring with the $\mathrm{Br}^{+}$cation. b) The ortho-subsituted product is formed. c) The metasubstituted product is formed. The full movie is available on YouTube. ${ }^{[58]}$ ficulty. However, the energy released is only about $40 \mathrm{~kJ} / \mathrm{mol}$. Students can, therefore, directly witness the stereoelectronic effect of a directing group at work. Of course, it is straightforward to investigate the effect of different directing groups and even the combination of them. For example, by replacing the amino group by a methyl substituent, students will find that the energy released upon formation of the para- and meta-substituted derivatives are about $170 \mathrm{~kJ} / \mathrm{mol}$ and $130 \mathrm{~kJ} / \mathrm{mol}$, respectively. Hence, it is immediately obvious that the $\mathrm{CH}_{3}$ group is a weaker ortho- and para-director compared to $\mathrm{NH}_{2}$.

This example is particularly interesting since the students will find that the creation of the $\sigma$-complex from the (active) electrophile and the aromatic system is thermodynamically favored. In most textbooks of organic chemistry, this step is usually presented to be unfavored (see, e.g. refs. [54-56]), even though there are counterexamples (see, e.g. ref. [57]).

The students will also find that upon adding the bromine cation to a carbon atom of the benzene ring, the bond between this carbon atom and the hydrogen atom will not break. Instead, the reaction will stop at the $\sigma$-complex. This clearly shows that the complete electrophilic aromatic substitution consists of two elementary steps. This stands in stark contrast to the previous example of an $\mathrm{S}_{\mathrm{N}} 2$ reaction, which features a single elementary step; the formation of a bond to the attacking group and the breaking of the bond to the leaving group happen in a concerted fashion. In an immersive interactive setting, students are able to experience, learn, and understand such concepts in an intuitive and descriptive way. A movie demonstrating this task can also be found on YouTube. ${ }^{[58]}$

\section{Conclusions and Outlook}

Modern approaches that rely on new computer hardware such as haptic (force-feedback) devices and virtual or augmented reality frameworks allow for immersive and hence more intuitive learning experiences. In this article, we showcased some example learning tasks typical for an undergraduate chemistry curriculum. In particular, we demonstrated how an embodiedlearning approach, making use of an interactive exploration and manipulation of the system by means of a haptic device, provides an illustrative and intuitive learning experience. This interactive exploration of the system is only possible by virtue of the advances made during the past years in the field of real-time quantum chemistry. ${ }^{[16]}$

However, we should emphasize that the present setting focuses on the electronic contribution to reactions and reaction energies. In other words, all reactions or chemical concepts dominated by electronic effects can be studied with our methodology. To also include entropic (rather than these enthalpic) contributions will require further developments in the real-time quantum chemistry framework (but such extensions are, in fact, already exploited in steered molecular dynamics settings ${ }^{[9,59]}$ which exploit Jarzynski's identity[60]).

It can be expected that such a learning setting can significantly improve the overall learning outcome. However, as a next step this needs to be rigorously investigated in extended user studies. Such work is currently being carried out in our laboratory within the Future Learning Initiative of ETH Zurich in collaboration with Prof. Manu Kapur and his group.

\section{Acknowledgments}

This work has been presented in a lecture with practical exercises for chemistry teachers at the fall meeting of the Swiss Chemical Society at the University of Zurich in September 2019.

Received: November 6, 2020

[1] T. N. Höffler, D. Leutner, Learn. Instr. 2007, 17, 722, https://doi.org/10.1016/j.learninstruc.2007.09.013 
[2] B. Tversky, J. B. Morrison, M. Betrancourt, Int. J. Hum. Comput. Stud. 2002, 57, 247, https://doi.org/10.1006/ijhc.2002.1017

[3] B. B. de Koning, H. K. Tabbers, Educ. Psychol. Rev. 2011, 23, 501, https://doi.org/10.1007/s10648-011-9173-8

[4] T. van Gog, F. Paas, N. Marcus, P. Ayres, J. Sweller, Educ. Psychol. Rev. 2009, 21, 21, https://doi.org/10.1007/s10648-008-9094-3

[5] W. D. Atkinson, K. E. Bond, G. L. Tribble, III, K. R. Wilson, Comput. and Graphics 1977, 2, 97, https://doi.org/10.1016/0097-8493(77)90009-7

[6] M. Ouh-young, D. V. Beard, F. P. Brooks, Jr., 'Force Display Performs Better than Visual Display in a Simple 6-D Docking Task', Proc. 1989 Int. Conf. on Robotics and Automation, 1989, pp 1462-1466.

[7] M. C. Surles, J. S. Richardson, D. C. Richardson, F. P. Brooks, Jr., Prot. Sci. 1994, 3, 198, https://doi.org/10.1002/pro.5560030205

[8] J. Leech, J. F. Prins, J. Hermans, IEEE Comput. Sci. Eng. 1996, 3, 38, https://doi.org/10.1109/99.556511

[9] J. E. Stone, J. Gullingsrud, K. Schulten, 'System for Interactive Molecular Dynamics Simulation', Proc. 2001 Symp. Interactive 3D Graphics, 2001, pp 191-194.

[10] A. Gillet, M. Sanner, D. Stoffler, A. Olson, Structure 2005, 13, 483, https://doi.org/10.1016/j.str.2005.01.009

[11] J. Murayama, H. Shimizu, C. S. Nam, H. Satoh, M. Sato, 'An Educational Environment for Chemical Contents with Haptic Interaction', 2007 Int. Conf. Cyberworlds (CW'07), 2007, pp 346-352.

[12] M. Limniou, D. Roberts, N. Papadopoulos, Comput. Educ. 2008, 51, 584.

[13] K. H. Marti, M. Reiher, J. Comput. Chem. 2009, 30, 2010, https://doi.org/10.1002/jcc.21201

[14] O. Delalande, N. Férey, G. Grasseau, M. Baaden, J. Comput. Chem. 2009, 30, 2375, https://doi.org/10.1002/jcc.21235

[15] M. Bosson, C. Richard, A. Plet, S. Grudinin, S. Redon, J. Comput. Chem. 2012, 33, 779, https://doi.org/10.1002/jcc. 22905

[16] M. P. Haag, M. Reiher, Int. J. Quantum Chem. 2013, 113, 8, https://doi.org/10.1002/qua.24336

[17] M. P. Haag, M. Reiher, Faraday Discuss. 2014, 169, 89, https://doi.org/10.1039/C4FD00021H

[18] M. P. Haag, A. C. Vaucher, M. Bosson, S. Redon, M. Reiher, ChemPhysChem 2014, 15, 3301, https://doi.org/10.1002/cphc.201402342

[19] Virtual and augmented reality immersive molecular simulations: general discussion, Faraday Discuss. 2014, 169, 143, https://doi.org/10.1039/C4FD90019G

[20] D. R. Glowacki, M. O'Connor, G. Calabró, J. Price, P. Tew, T. Mitchell, J. Hyde, D. P. Tew, D. J. Coughtrie, S. McIntosh-Smith, Faraday Discuss. 2014, 169, 63, https://doi.org/10.1039/c4fd00008k

[21] N. Luehr, A. G. B. Jin, T. J. Martínez, J. Chem. Theory Comput. 2015, 11, 4536, https://doi.org/10.1021/acs.jctc.5b00419

[22] M. Norrby, C. Grebner, J. Eriksson, J. Boström, J. Chem. Inf. Model. 2015, 55, 2475, https://doi.org/10.1021/acs.jcim.5b00544

[23] A. Salvadori, G. D. Frate, M. Pagliai, G. Mancini, V. Barone, Int. J. Quantum Chem. 2016, 116, 1731, https://doi.org/10.1002/qua.25207

[24] M. Zheng, M. P. Waller, J. Mol. Graphics Modell. 2017, 73, 18, https://doi.org/10.1016/j.jmgm.2017.01.019

[25] M. O'Connor, H. M. Deeks, E. Dawn, O. Metatla, A. Roudaut, M. Sutton, L. M. Thomas, B. R. Glowacki, R. Sage, P. Tew, M. Wonnacott, P. Bates, A. J. Mulholland, D. R. Glowacki, Sci. Adv. 2018, 4, eaat2731, https://doi.org/10.1126/sciadv.aat2731

[26] C. Müller, M. Krone, M. Huber, V. Biener, D. Herr, S. Koch, G. Reina, D. Weiskopf, T. Ertl, J. Integr. Bioinform. 2018, 15, 20180005, https://doi.org/10.1515/jib-2018-0005

[27] L. J. Kingsley, V. Brunet, G. Lelais, S. McCloskey, K. Milliken, E. Leija, S. R. Fuhs, K. Wang, E. Zhou, G. Spraggon, J. Mol. Graphics Modell. 2019, 89, 234, https://doi.org/10.1016/j.jmgm.2019.03.010

[28] R. J. García-Hernández, D. Kranzmüller, Comput. Phys. Commun. 2019 237, 230, https://doi.org/10.1016/j.cpc.2018.11.013

[29] J. B. Ferrell, J. P. Campbell, D. R. McCarthy, K. T. McKay, M. Hensinger, R. Srinivasa, X. Zhao, A. Wurthmann, J. Li, S. T. Schneebeli, J. Chem. Educ. 2019, 96, 1961, https://doi.org/10.1021/acs.jchemed.9b00036

[30] R. Dai, J. A. Laureanti, M. Kopelevich, P. L. Diaconescu, J. Chem. Educ. 2020, 97, 3647, https://doi.org/10.1021/acs.jchemed.0c00469

[31] D. G. Doak, G. S. Denyer, J. A. Gerrard, J. P. Mackay, J. R. Allison, Prot. Sci. 2020, 29, 157, https://doi.org/10.1002/pro.3752

[32] H. A. Gandhi, S. Jakymiw, R. Barrett, H. Mahaseth, A. D. White, J. Chem. Educ. 2020, https://doi.org/10.1021/acs.jchemed.9b01161.
[33] B. Sanii, J. Chem. Educ. 2020, 97, 253, https://doi.org/10.1021/acs.jchemed.9b00577

[34] https://cadd.ethz.ch/education/hololense.html (accessed November 04, 2020).

[35] C. Dede, M. C. Salzman, R. B. Loftin, D. Sprague, 'Multisensory Immersion as a Modeling Environment for Learning Complex Scientific Concepts', in Eds. W. Feurzeig, N. Roberts, 'Modeling and Simulation in Science and Mathematics Education', 1999, pp 282-319, https://doi.org/10.1007/978-1-4612-1414-4_12

[36] J. Trindade, C. Fiolhais, L. Almeida, Br. J. Educ. Technol. 2002, 33, 471, https://doi.org/10.1111/1467-8535.0028

[37] S. J. Bennie, K. E. Ranaghan, H. Deeks, H. E. Goldsmith, M. B. O’Connor, A. J. Mulholland, D. R. Glowacki, J. Chem. Educ. 2019, 96, 2488, https://doi.org/10.1021/acs.jchemed.9b00181

[38] F. M. Fung, W. Y. Choo, A. Ardisara, C. D. Zimmermann, S. Watts, T. Koscielniak, E. Blanc, X. Coumoul, R. Dumke, J. Chem. Educ. 2019, 96 , 382, https://doi.org/10.1021/acs.jchemed.8b00728

[39] Z. A. Jiménez, 'Technology Integration in Chemistry Education and Research (TICER)', American Chemical Society, 2019, pp 31-52.

[40] N. Ali, S. Ullah, J. Chem. Educ. 2020, 97, 3563, https://doi.org/10.1021/acs.jchemed.0c00185

[41] J. Minogue, M. G. Jones, Rev. Educ. Res. 2006, 76, 317, https://doi.org/10.3102/00346543076003317

[42] P. Bivall, S. Ainsworth, L. A. E. Tibell, Sci. Educ. 2011, 95, 700, https://doi.org/10.1002/sce.20439

[43] Z. C. Zacharia, Educ. Res. Rev. 2015, 16, 116 https://www.learntechlib.org/p/197385/

[44] B. I. Edwards, K. S. Bielawski, R. Prada, Virtual Real. 2019, 23, 363, https://doi.org/10.1007/s10055-018-0345-4

[45] 'Haptic Rendering', Eds. M. C. Lin, M. Otaduy, CRC Press, 2008.

[46] H. R. Malone, O. N. Syed, M. S. Downes, A. L. D'Ambrosio, D. O. Quest, M. G. Kaiser, Neurosurg. 2010, 67, 1105 , https://doi.org/10.1227/NEU.0b013e3181ee46d0

[47] D. D. Ruikar, R. S. Hegadi, K. C. Santosh, J. Med. Syst. 2018, 42, 168 https://doi.org/10.1007/s10916-018-1019-1

[48] F. Dachille, H. Qin, A. Kaufman, J. El-Sana, 'Haptic Sculpting of Dynamic Surfaces', Proc. 1999 Symp. Interactive 3D Graphics, 1999, p 103110.

[49] A. C. Vaucher, M. P. Haag, M. Reiher, J. Comput. Chem. 2016, 37, 805, https://doi.org/10.1002/jcc. 24268

[50] https://scine.ethz.ch/download/interactive (accessed November 04, 2020).

[51] https://www.samson-connect.net/ (accessed November 04, 2020).

[52] A. H. Mühlbach, A. C. Vaucher, M. Reiher, J. Chem. Theory Comput. 2016, 12, 1228, https://doi.org/10.1021/acs.jctc.5b01156

[53] https://youtu.be/imYmcWboj8o (accessed November 04, 2020).

[54] K. P. C. Vollhardt, N. E. Schore, 'Organic Chemistry: Structure and Function', 4th ed., W. H. Freeman and Company, 2003.

[55] P. A. Sykes, 'Guidebook to Mechanism in Organic Chemistry', 6th ed. Longman Group UK Ltd., 1986.

[56] F. Badea, 'Reaction Mechanisms in Organic Chemistry', Abacus Press, 1977.

[57] F. A. Carey, R. J. Sundberg, 'Advanced Organic Chemistry', 5th ed., Springer, 2007.

[58] https://youtu.be/cnoodBFfcVM (accessed November 04, 2020).

[59] M. O. Jensen, S. Park, E. Tajkhorshid, K. Schulten, Proc. Natl. Acad. Sci. U.S.A. 2002, 99, 6731, https://doi.org/10.1073/pnas.102649299

[60] C. Jarzynski, Phys. Rev. Lett. 1997, 78, 2690, https://doi.org/10.1103/PhysRevLett.78.2690

\section{License and Terms}

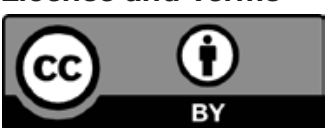

This is an Open Access article under the terms of the Creative Commons Attribution License CC BY 4.0. The material may not be used for commercial purposes.

The license is subject to the CHIMIA terms and conditions: (http:// chimia.ch/component/sppagebuilder/?view=page\&id=12).

The definitive version of this article is the electronic one that can be found at https://doi.org/10.2533/chimia.2021.45 